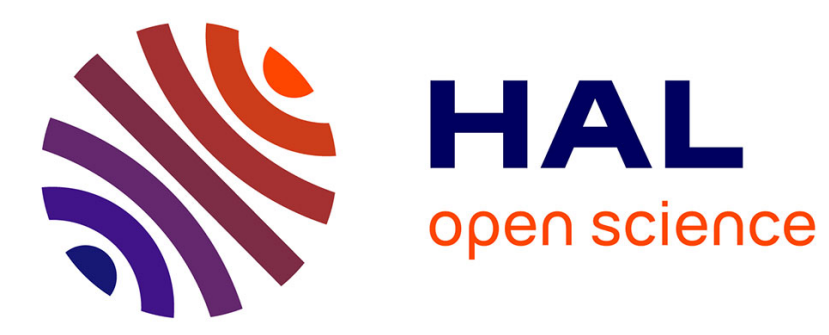

\title{
Enhanced Terahertz Imaging of Small Forced Delamination in Woven Glass Fibre-reinforced Composites with Wavelet De-noising
}

\author{
Junliang Dong, A. Locquet, D. S Citrin
}

\section{- To cite this version:}

Junliang Dong, A. Locquet, D. S Citrin. Enhanced Terahertz Imaging of Small Forced Delamination in Woven Glass Fibre-reinforced Composites with Wavelet De-noising. Journal of Infrared, Millimeter and Terahertz Waves, 2016, 37 (3), pp.289-301. 10.1007/s10762-015-0226-9 . hal-03048526

\author{
HAL Id: hal-03048526 \\ https://hal.science/hal-03048526
}

Submitted on 9 Dec 2020

HAL is a multi-disciplinary open access archive for the deposit and dissemination of scientific research documents, whether they are published or not. The documents may come from teaching and research institutions in France or abroad, or from public or private research centers.
L'archive ouverte pluridisciplinaire HAL, est destinée au dépôt et à la diffusion de documents scientifiques de niveau recherche, publiés ou non, émanant des établissements d'enseignement et de recherche français ou étrangers, des laboratoires publics ou privés. 


\title{
Enhanced Terahertz Imaging of Small Forced Delamination in Woven Glass Fiber-reinforced Composites with Wavelet De-noising
}

\author{
Junliang Dong • Alexandre Locquet . \\ D. S. Citrin
}

Received: date / Accepted: date

\begin{abstract}
Terahertz $(\mathrm{THz})$ reflection imaging is applied to characterize a woven glass fiber-reinforced composite laminate with a small region of forced delamination. The forced delamination is created by inserting a disk of $25 \mu \mathrm{m}$ thick Upilex film, which is below the $\mathrm{THz}$ axial resolution, resulting in one featured echo with small amplitude in the reflected $\mathrm{THz}$ pulses. Low-amplitude components of the temporal signal due to ambient water vapor produce features of comparable amplitude with features associated with the $\mathrm{THz}$ pulse reflected off the interfaces of the delamination, and suppress the contrast of $\mathrm{THz} \mathrm{C}$ - and B-scans. Wavelet shrinkage de-noising is performed to remove water-vapor features, leading to enhanced $\mathrm{THz} \mathrm{C}$ - and B-scans to locate the delamination in three dimensions with high contrast.
\end{abstract}

Keywords $\mathrm{THz}$ imaging · Wavelet de-noising · Forced delamination · Woven glass fiber-reinforced composite $\cdot$ Water vapor effects

\footnotetext{
Junliang Dong

Georiga Tech-CNRS, UMI2958, Georgia Tech Lorraine, Metz 57070, France

School of Electrical and Computer Engineering, Georgia Institute of Technology, Atlanta, GA, 30332 USA

E-mail: junliang.dong@gatech.edu

Alexandre Locquet

Georiga Tech-CNRS, UMI2958, Georgia Tech Lorraine, Metz 57070, France

School of Electrical and Computer Engineering, Georgia Institute of Technology, Atlanta, GA, 30332 USA

E-mail: alexandre@gatech.edu

D. S. Citrin

Georiga Tech-CNRS, UMI2958, Georgia Tech Lorraine, Metz 57070, France

School of Electrical and Computer Engineering, Georgia Institute of Technology, Atlanta, GA, 30332 USA

E-mail: david.citrin@ece.gatech.edu
} 


\section{Introduction}

Terahertz $(\mathrm{THz})$ imaging, which can provide a non-invasive, non-contact and non-ionizing modality to characterize various non-metallic materials, is emerging as a promising nondestructive evaluation (NDE) technique for fiber-reinforced composites. Fiber-reinforced composites combine the merits of high strength and low weight, offering an alternative to conventional structural materials for various applications including aerospace, automotive, renewable energy and marine. $\mathrm{THz}$ imaging was first applied to the NDE of fiber-reinforced composites in 2006 [1], and has been explored extensively since [2]. For glass fiberreinforced composites, $\mathrm{THz}$ waves can be utilized to detect both surface and underlying defects, including mechanical/heat damage [3], voids [4], delaminations [5], intrusions and moisture contamination [6]. Compared with ultrasonic C-scans, the most widely implemented NDE technique for composites in industry, THz imaging can provide higher spatial resolution for characterizing glass fiber-reinforced composites in three dimensions [5]. Moreover, THz waves can also be applied to measure the optical parameters [7] and fiber orientation [8] in glass fiber-reinforced composites. Besides glass fiber-reinforced composites, composites reinforced by other non-conductive fibers, such as ultra-high molecular weight polyethylene(UHMWPE) composites, have also been explored with $\mathrm{THz}$ imaging for characterization of delaminations $[9,10]$. For carbon fiberreinforced composites, due to the conductivity of carbon fibers, $\mathrm{THz}$ imaging has largely been applied to detect surface or near-surface features, such as impact-induced matrix cracking [11]. Although the conductivity of carbon fibers hinders $\mathrm{THz}$ applications at depth, the anisotropic conductivity of unidirectional carbon fibers can also in cases enable the extraction of important information. For example, in Ref. [12], THz imaging is applied to a hybrid fiberreinforced composite containing glass-fibers/epoxy and carbon-fibers/epoxy plies with a cross-ply stack pattern. Impact-induced intra- and inter-laminar damages at the same inter face can be differentiated via polarization-resolved $\mathrm{THz}$ imaging due to the strong conductivity anisotropy in the unidirectional nature of the carbon fibers.

In the $\mathrm{THz}$ frequency region, many polar gases possess pronounced rotational transitions, leading to numerous spectral resonances. Water vapor, as the third most abundant gas in the terrestrial atmosphere, is known to have many rotational resonances in the $\mathrm{THz}$ frequency region [13]. Therefore, $\mathrm{THz}$ imaging of a sample in the open air results in a combination of the sample's spectral features and ambient water-vapor resonances. In the time domain, this results in ringing - $\mathrm{THz}$ fluctuations - after the dominant $\mathrm{THz}$ pulse [14]. Provided the features of interest are sufficiently large, these fluctuations can be ignored. However, when the real features from the sample are of comparable amplitude, they may be obscured by features associated with water vapor, leading to $\mathrm{THz}$ images with low contrast. Clearly, ridding the system of ambient water vapor, such as by purging with dry nitrogen, can suppress these fluctuations; however, it is not always possible to enclose the entire $\mathrm{THz}$ beam path in many practical laboratory and field applications. Several numerical ap- 
proaches have been studied to remove the water-vapor fluctuations, including adaptive deconvolution [14], absorbance spectrum fitting [15], artificial neural networks [16], and wavelet de-noising [17].

In this study, THz imaging is applied to characterize small forced delamination in woven glass fiber-reinforced composites. The forced delamination is created by inserting Upilex film with thickness only about $25 \mu \mathrm{m}$. THz echoes reflected from the front and rear interfaces of the forced delamination cannot be readily separated in the raw signal, leading to only one distinguishable echo in time associated with the delamination. The amplitude of this featured echo is small, and is comparable to the fluctuations resulting from the ambient water vapor. Wavelet shrinkage de-noising is performed here to suppress these fluctuations and to obtain enhanced $\mathrm{THz} \mathrm{C}$ - and B-scan images for characterizing the delamination in three dimensions.

\section{Theoretical Background}

\section{$2.1 \mathrm{THz}$ Time-of-flight Waveform}

In the case of a sample with forced delamination, the temporal reflected $\mathrm{THz}$ pulses can be estimated, based on considerations shown schematically in Fig. 1. For normal incidence on an interface between two media with refractive indices $n_{i}$ and $n_{j}$, the amplitude reflection coefficient $r_{i j}$ and transmission coefficient $t_{i j}$ are

$$
\begin{aligned}
& r_{i j}=\frac{n_{i}-n_{j}}{n_{i}+n_{j}} \\
& t_{i j}=\frac{2 n_{i}}{n_{i}+n_{j}}
\end{aligned}
$$

In our study, the subscripts $(i, j)$ for different media are: $a$-air, $g$-glass fiberreinforced composites and $d$-forced delamination.

The reflected $\mathrm{THz}$ time-of-flight waveform is composed of the echoes off interfaces between various media. In principle, the temporal separation between echoes is proportional to the distance between interfaces (the thickness of the forced-delamination). And the minimum distance discernable from the signal, viz. the axial resolution $d_{m i n}$, is

$$
d_{\text {min }}=\frac{c \Delta T}{2 n_{d}}
$$

where $c$ is the in-vacuo speed of light, $\Delta T$ is the temporal width of the $\mathrm{THz}$ pulse, and $n_{d}$ is the group refractive index of the medium within the forced delamination. When the forced-delamination thickness is larger than the axial resolution, two distinct $\mathrm{THz}$ echoes from the consecutive interfaces are well separated in time; otherwise, the two $\mathrm{THz}$ echoes cannot be easily distinguished. Therefore, in our case with thin delaminations below the axial resolution, the 
reflected $\mathrm{THz}$ waveform $E_{T H z}(t)$ is expected to be composed of three clear features,

$$
E_{T H z}(t)=E_{1 s t}(t)+E_{2 n d}(t)+E_{3 r d}(t)
$$

The first and third terms, $E_{1 s t}(t)$ and $E_{3 r d}(t)$, correspond to Fresnel reflections at the bottom and top surfaces of the sample. And the second term echo, $E_{2 n d}(t)$, is the superposition of the two echoes returned from the interfaces associated with the delamination. In our case, we neglect the negligible dispersion in the relevent spectral range, so this featured echo $E_{2 n d}(t)$ can be expressed based on the incident $\mathrm{THz}$ pulse $E_{\text {in }}(t)$,

$$
\begin{aligned}
E_{2 n d}(t) & =E_{g d}(t)+E_{d g}(t) \\
& =t_{a g} r_{g d} t_{g a} E_{i n}\left(t-\frac{2 n_{g} d^{\prime}}{c}\right) e^{-2 \alpha d^{\prime}} \\
& +t_{a g} t_{g d} r_{d g} t_{d g} t_{g a} E_{i n}\left(t-\frac{2 n_{g} d^{\prime}}{c}-\frac{2 n_{d} d}{c}\right) e^{-2 \alpha^{\prime} d^{\prime}} e^{-2 \alpha d}
\end{aligned}
$$

with $d^{\prime}$ the distance between the delamination and the surface of the sample, $d$ the thickness of the delamination, $\alpha^{\prime}$ and $\alpha$ the absorption coefficients in the glass fiber-reinforced composites and in the forced delamination. Because the signs of the reflection coefficients $\left(r_{g d}\right.$ and $\left.r_{d g}\right)$ at the interfaces between the glass fiber-reinforced composite and the delamination are opposite, the two echoes $E_{g d}(t)$ and $E_{d g}(t)$ will tend to cancel with each other, leading to the small amplitude of the associated signal $E_{2 n d}(t)$. Moreover, the signal amplitude decreases with decreasing delamination thickness [18].

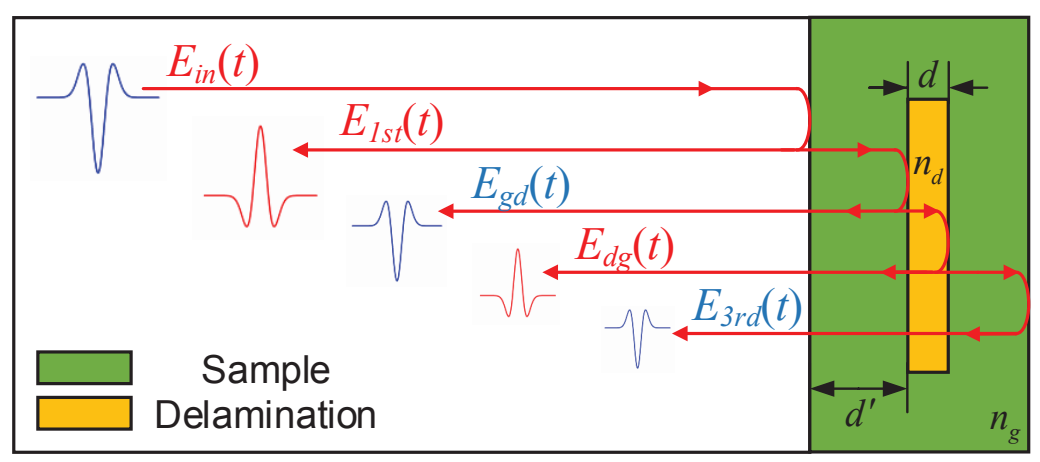

Fig. 1 Estimated reflected $\mathrm{THz}$ pulses in region with delamination indicating the observed features associated with reflections off various interfaces. 


\subsection{Wavelet de-noising}

Wavelet shrinkage is a signal de-noising technique based on the idea of thresholding the wavelet coefficients. Mittleman [19] first suggested the use of wavelet transforms for $\mathrm{THz}$ signal processing because the $\mathrm{THz}$ pulse is similar in form to common wavelet basis functions. Compared with the general discrete wavelet transform $(D W T)$, the stationary wavelet transform $(S W T)$ is never sub-sampled and instead up-sampled at each level of decomposition. $S W T$ decomposes a $1 D$ signal $x(n)$ into the approximation coefficients vector $c A_{k, l}$ and detail coefficients $c D_{k, l}$ by convolving with a low-pass filter $\Psi$ and a high-pass filter $\Phi$ along the temporal axis at each level [20], shown in Fig. 2. Wavelet coefficients with small absolute values can be considered as noise, and wavelet coefficients with large absolute values are regarded as the main featured information of the signal. Removing the small absolute value coefficients by thresholding and then reconstructing the signal is expected to produce a signal in which the contribution of noise has been reduced.

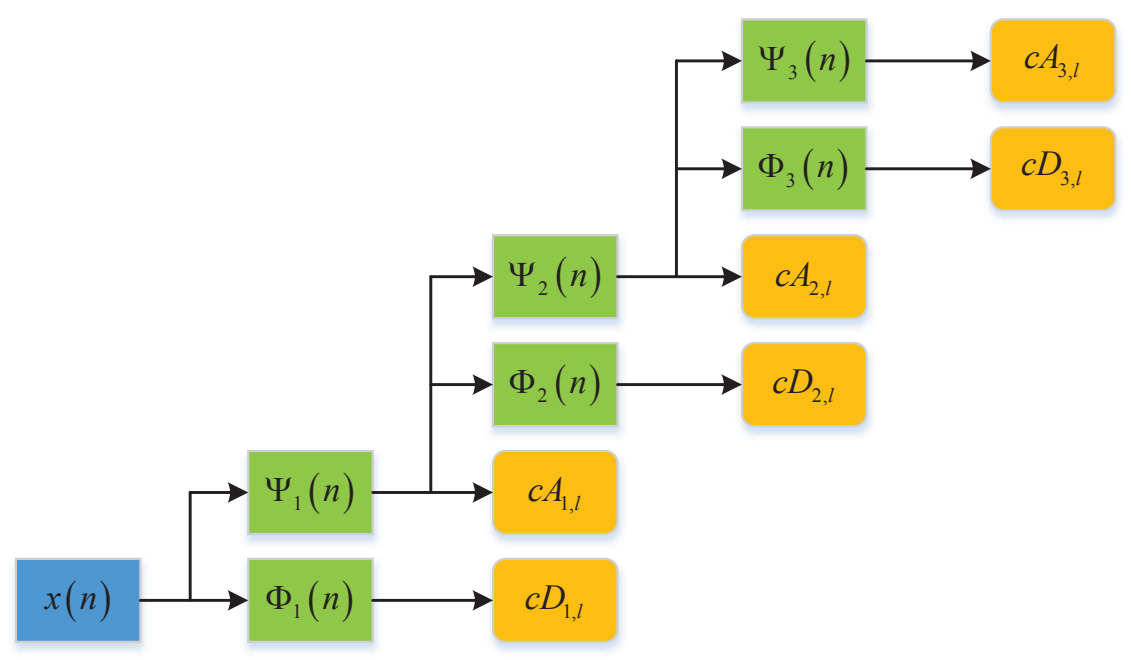

Fig. 2 Schematic level-3 decomposition of a signal with stationary wavelet transform $(S W T)$.

In general, wavelet shrinkage de-noising with soft thresholding is performed via the following procedure [20,21]:

1. Determine the wavelet coefficients by taking the $S W T$,

$$
[c A, c D]=S W T(x(n))
$$

2. Calculate the threshold value $T$,

$$
T=\sigma \sqrt{2 \log _{e} N}
$$


where $\sigma$ is the noise level and $N$ is the number of sampling points.

3 . Threshold the wavelet coefficients $c D_{k, l}$ with solft-thresholding

$$
c \hat{D}_{k . l}= \begin{cases}c D_{k, l}-T & c D_{k, l} \geq T, \\ c D_{k, l}+T & c D_{k, l} \leq-T, \\ 0 & \left|c D_{k, l}\right|<T .\end{cases}
$$

4. Perform the inverse stationary wavelet transform $(I S W T)$ to recover the time domain signal $\hat{x}(n)$,

$$
\hat{x}(n)=I S W T([c A, c \hat{D}])
$$

\section{Sample and Experiment Setup}

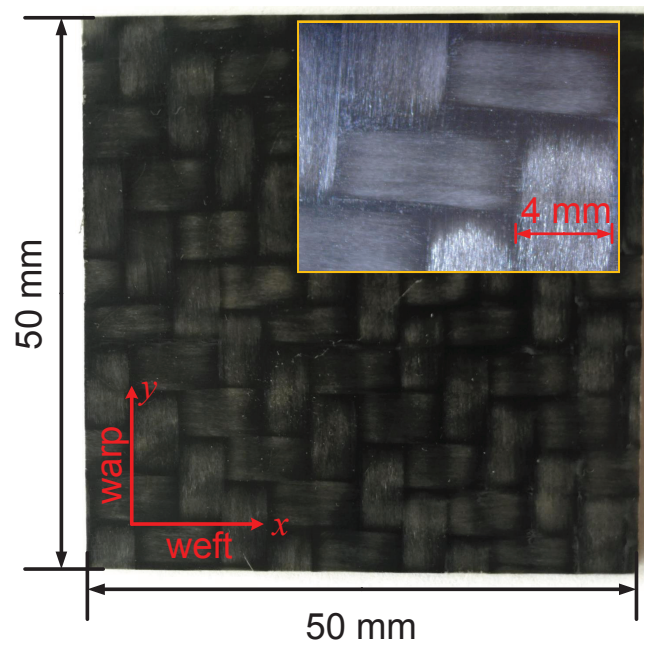

Fig. 3 The photograph of the woven E-glass fiber reinforced nylon matrix laminate. Inset: Amplification of the photograph to show the size of the fiber bundles.

A woven $(2 \times 2$ twill weave $)$ E-glass fiber reinforced nylon matrix laminate, shown in Fig. 3, is employed in this study. The dimensions of the laminate are $50 \mathrm{~mm}$ (length) $\times 50 \mathrm{~mm}$ (width) $\times 1.54 \mathrm{~mm}$ (thickness), and the surfaces are flat. The laminate is composed of three glass fabric layers stacked as $\left(0^{\circ} / 90^{\circ}\right)$ with fiber volume fraction about $50 \mathrm{vol} \%$. The size of the fiber bundles is 1200 tex (weight in grams per kilometer of yarns), shown in the inset of Fig. 3. Delamination was introduced between the first and second fabric layer by incorporating a Upilex (ultra-high heat-resistant polymide) release film disk with thickness about $25 \mu \mathrm{m}$ and diameter about $12 \mathrm{~mm}$ prior to consolidation of the laminate. 
A $\mathrm{THz}$ time-domain spectroscopy (TDS) and imaging system (Teraview TPS Spectra 3000) is employed in this study, shown schematically in Fig. 4. The GaAs photoconductive antenna is excited by an ultrafast (femtosecond) laser to produce roughly single-cycle $\mathrm{THz}$ pulses with bandwidth extending from $60 \mathrm{GHz}$ to $3 \mathrm{THz}$. The ultrafast laser used here is an Er-doped fiber laser that emits $780 \mathrm{~nm}$ pulses with sub-100 femtosecond pulse duration at a repetition rate of $100 \mathrm{MHz}$ and has an average output power in excess of $65 \mathrm{~mW}$. Coherent detection of the $\mathrm{THz}$ radiation is performed in a similar photoconductive antenna circuit. By gating the photoconductive gap with a femtosecond pulse synchronized to the $\mathrm{THz}$ emission, a current proportional to the $\mathrm{THz}$ electric field is measured. By varying the optical path length, the reflected $\mathrm{THz}$ time-domain pulse can be sampled, resulting in both temporal amplitude and phase information.

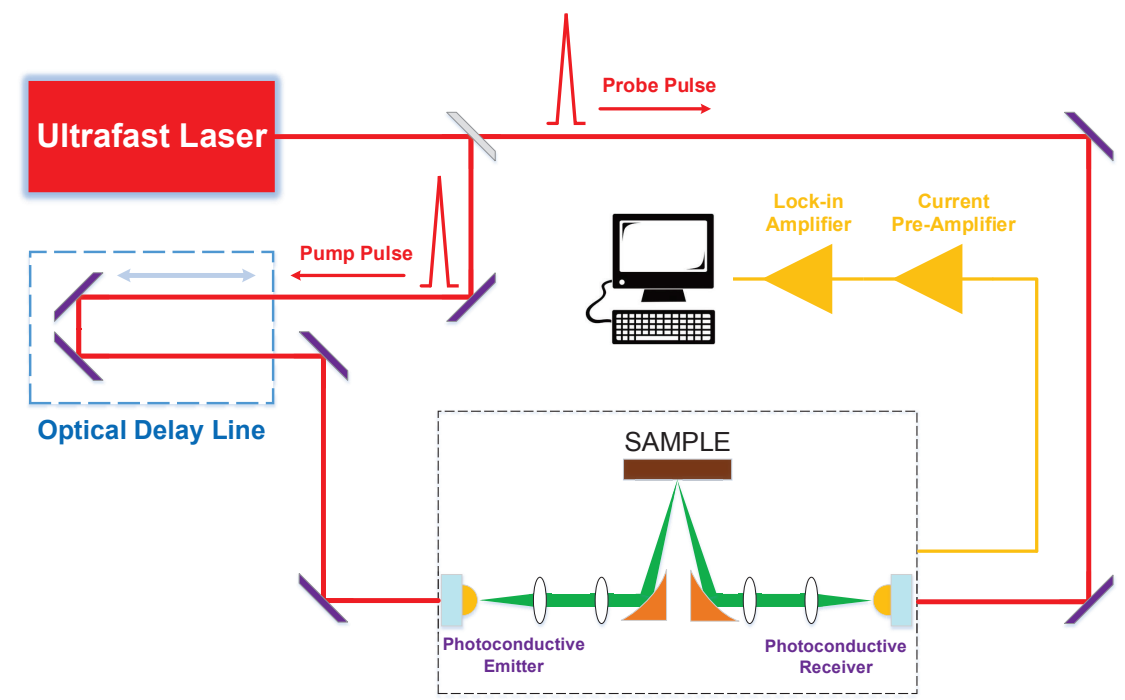

Fig. 4 Schematic diagram of THz time-domain spectroscopy (TDS) and imaging system in reflection mode.

$\mathrm{THz}$ imaging is performed in reflection mode at almost normal incidence from the bottom surface of the sample. The polarization of the THz beam is in the $y$ direction, which is parallel to the warp direction of the sample. Experiments were carried out in an air-conditioned laboratory with temperature at $22^{\circ} \mathrm{C}$. The humidity in the laboratory was $48 \%$, but no provision was made for backfilling with dry nitrogen. Before imaging the sample, a $\mathrm{THz}$ reference signal was recorded by setting a metal plate at the sample position. The reference $\mathrm{THz}$ pulse and spectrum are shown in Fig. 5. Pronounced fluctuations behind the main $\mathrm{THz}$ pulse due to atmospheric water-vapor absorption can be clearly observed. The sample is raster-scanned by a set of motorized stages 
moving in the $x$ and $y$ directions with a $0.2 \mathrm{~mm}$ spatial step over a $45 \mathrm{~mm} \times$ $45 \mathrm{~mm}$ region of the sample. Each recorded reflected $\mathrm{THz}$ pulse contains 4096 data points and the signal is averaged over 5 shots per pixel.
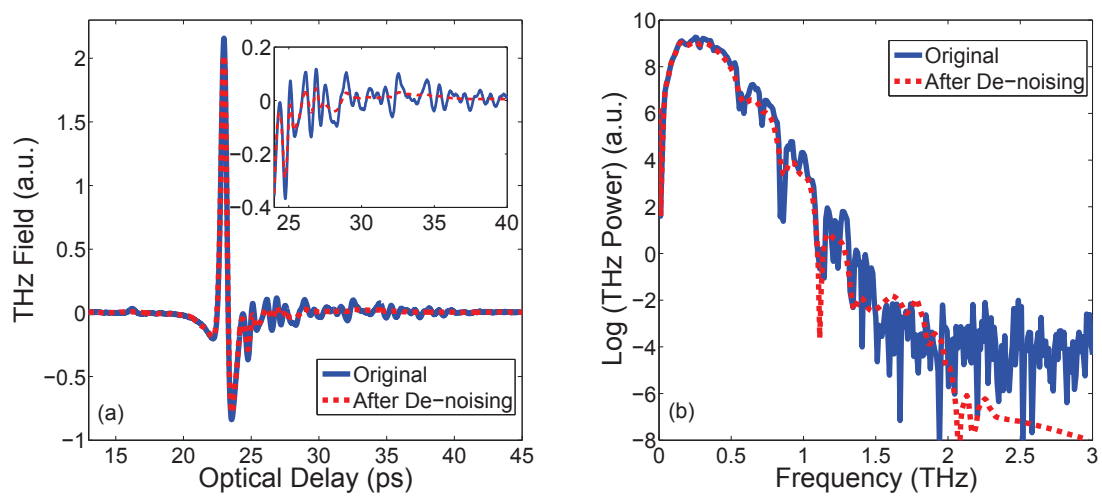

Fig. $5 \mathrm{THz}$ reference signal with and without wavelet de-noising in (a) time domain and (b) frequency domain. The inset in (a) shows the fluctuations in the signal due to ambient water vapor.

\section{Results and Discussion}

4.1 Identifying the water-vapor effect in typical signal from the sample

Before performing wavelet shrinkage de-noising to the reflected $\mathrm{THz}$ pulses, we implemented a numerical parameter fitting method based on multiple regression analysis [22] to make sure that the echoes between the featured echoes corresponding to the bottom and top surfaces in the reflected $\mathrm{THz}$ signals are mainly due to the water vapor. A typical measured $\mathrm{THz}$ waveform without delamination, shown in Fig. 6, is considered as the objective function. Echoes reflected from the bottom and top surfaces of the samples can be clearly identified in this reflected waveform. (Multiple reflections are small and correspond to features in the reflected $\mathrm{THz}$ pulse that occur at later times.) A model function is defined to simulate the objective function as

$$
E_{\text {model }}=a E_{\text {in }}(t-\Delta t)
$$

where $a$ and $\Delta t$ are the regression parameters. We consider the original $\mathrm{THz}$ reference signal shown in Fig. 5 as the incident $\mathrm{THz}$ pulse $E_{\text {in }}(t)$ and ignore the echo from the top surface in this simulation. In short, we use an amplitude-decreased and time-shifted reference signal to match the first echo in the measured signal. Initial values should be carefully set so that the regression analysis can converge to a characteristic global minimum. Then multiple 
regression analysis with least-squares minimization is performed to estimate the regression parameters, and the results are shown in Fig. 6. Signals between the Fresnel reflections from the bottom and top surfaces of the sample match quite well with the model function with regression parameters. The coefficient of determination $R^{2}$ is also used here to provide information about the goodness of fit. In the time slice between 24 and $34 \mathrm{ps}$, the coefficient of determination equals to 0.9827 , which demonstrates that these features mainly originate from the water vapor.

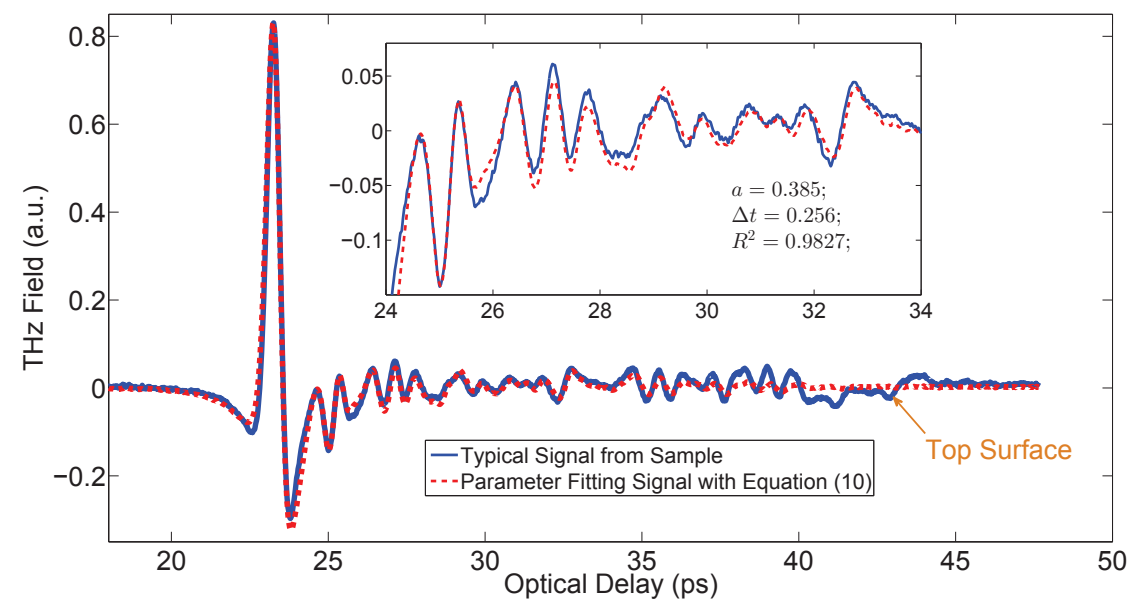

Fig. 6 Multiple parameter regress analysis of measured $\mathrm{THz}$ waveform without delamination to identify the water-vapor effect.

It should be noted that featured echoes corresponding to the reflections from the internal interfaces between the fabric layers cannot be identified in the typical $\mathrm{THz}$ reflected waveform. Due to the complex internal structure of the woven fiber-reinforced composites with big size of fiber bundles, the echoes reflected from the internal interfaces are very small (comparable or even smaller than the features introduced by the water vapor), and these small echoes are immersed in the reflected waveform. This is also the reason why a small mismatch can be found in Fig. 6 in the time slice between 27 and $29 \mathrm{ps}$, which corresponds to the location of the first internal interface between fabric layers.

\section{$4.2 \mathrm{THz} \mathrm{C}$ - and B-scans with wavelet denoising}

Next, wavelet shrinkage de-noising is performed to process the reference signal and the $\mathrm{THz}$ pulses in regions with and without delamination, respectively. In this study, we choose the symlet (sym4) wavelets, which are a modified 

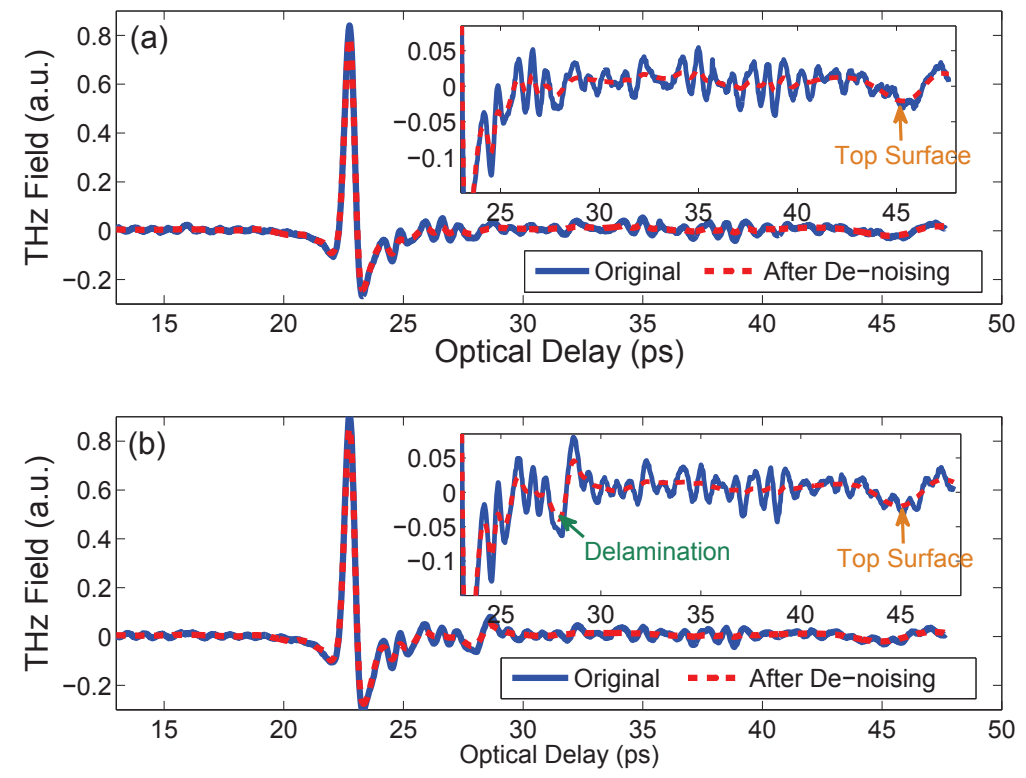

Fig. 7 Typical reflected $\mathrm{THz}$ waveforms with and without wavelet de-noising in the region (a) without and (b) with delamination.

form of Daubechies wavelets. A maximum level of 7 is used for the wavelet decomposition as no significant improvement was observed for higher levels to justify the extra computational expense. The position of the noise interval is set to the region of the fluctuations just after the main $\mathrm{THz}$ pulse in the original reference signal, which locates in the temporal region between 24 and $40 \mathrm{ps}$.

The time-domain $\mathrm{THz}$ reference signal and its spectrum after wavelet denoising are shown in Fig. 5 for comparison. In time domain [Fig. 5(a)], most of the water-vapor fluctuations are removed without losing much energy in the main $\mathrm{THz}$ pulse. In the frequency domain [Fig. 5(b)], the major water-vapor absorption dips are successfully suppressed.

To provide B- and C-scans, we processed the $\mathrm{THz}$ raw data obtained by raster-scanning with wavelet de-noising for each pixel. Typical reflected $\mathrm{THz}$ pulses without and with delamination and the comparison before and after wavelet de-noising are shown in Fig. 7. The echo associated with delamination [Fig. 7 (b)] can be located with small amplitude comparable to the level of fluctuations. After wavelet de-noising, most of the fluctuations between the echoes from the bottom and top surfaces are successfully removed. However, the features associated with the structure, including the echoes from the delamination and the bottom and top surfaces of the sample, are evident. In this 
way, the signal-to-noise ratio is also be increased providing higher contrast for $\mathrm{THz} \mathrm{C}$ - and B-scans.
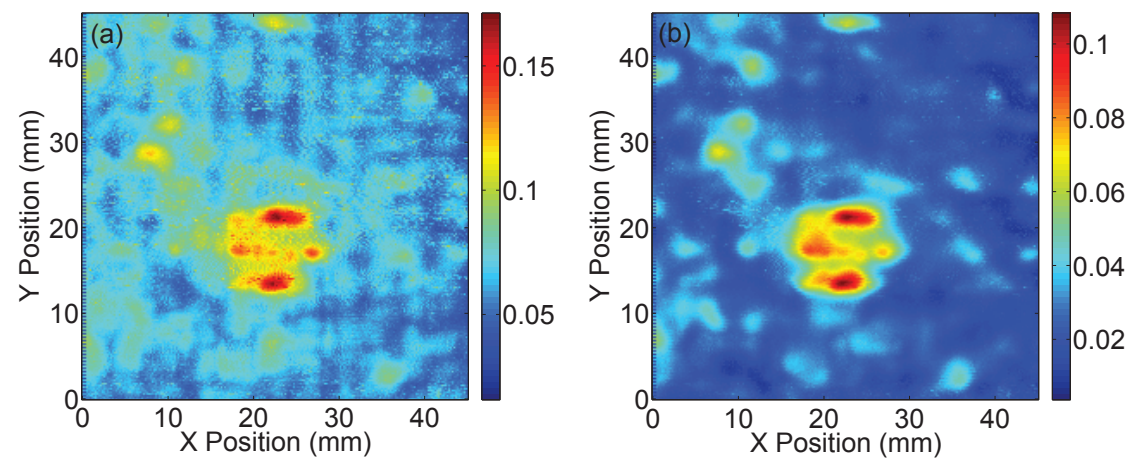

Fig. $8 \mathrm{THz} \mathrm{C}$-scan images based on $\mathrm{THz}$ waveforms (a) without and (b) with wavelet de-noising.
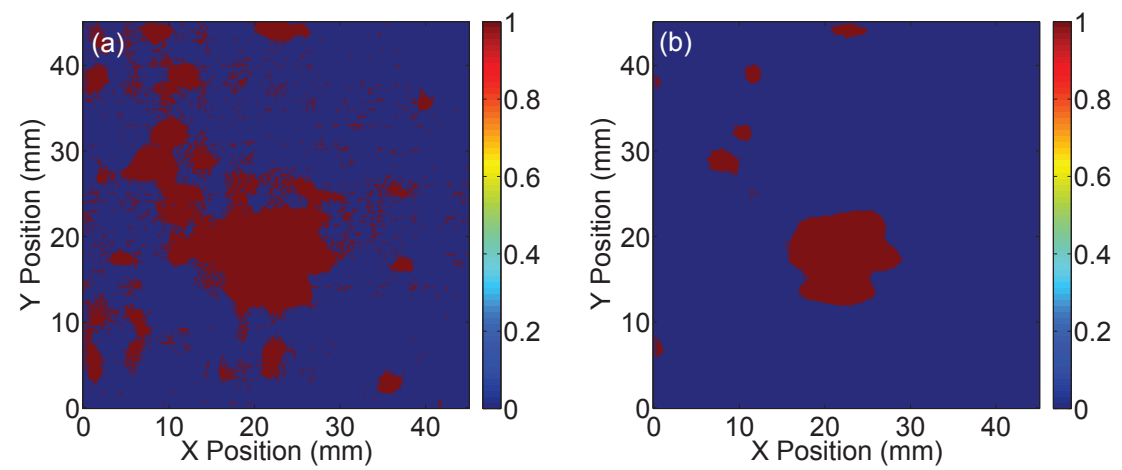

Fig. 9 Binary $\mathrm{THz}$ C-scan images based on $\mathrm{THz}$ waveforms (a) without and (b) with wavelet de-noising.

$\mathrm{THz}$ C-scans without and with wavelet de-noising are shown in Fig. 8. The contrast mechanism chosen here is the difference between the maximum and minimum values of the reflected $\mathrm{THz}$ signal within a selected time slice between 27 and $29 \mathrm{ps}$, which corresponds to the location of the delamination. $\mathrm{THz}$ C-scans with wavelet de-noising show higher contrast with regard to the delamination area and better enable us to differentiate the edge of the delamination from the background. This delamination is of roughly circular shape corresponding to the $12 \mathrm{~mm}$ diameter Upilex film disk. Due to the big size of the fiber bundles, the thin Upilex film inside is deformed and the woven fiber fabric produces the vague grid pattern in the C-scan. We can further 
apply the $50 \%$ rule [23] to obtain binary $\mathrm{THz}$ C-scans, which only consists two areas, denoting the suspected delamination area as ' 1 ', and others as ' 0 '. This method locates the pixel with the highest value in the C-scan and assigns to it a value of $100 \%$, then colors all pixels red that have a value of at least $50 \%$ of the maximum. Binary $\mathrm{THz} \mathrm{C}$-scans are frequently used for sizing delaminations [5]. The binary $\mathrm{THz}$ C-scans based on the data without and with wavelet denoising are shown in Fig. 9. Despite the presence of small artifacts, the binary $\mathrm{THz}$ C-scan based on the processed data provides a significantly better image to locate and size the delamination.
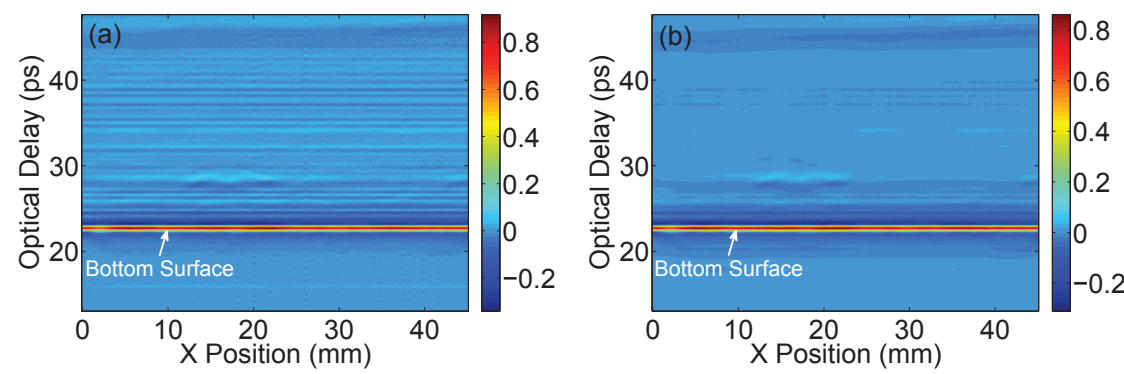

Fig. $10 \mathrm{THz}$ B-scan images based on $\mathrm{THz}$ waveforms (a) without and (b) with wavelet de-noising.
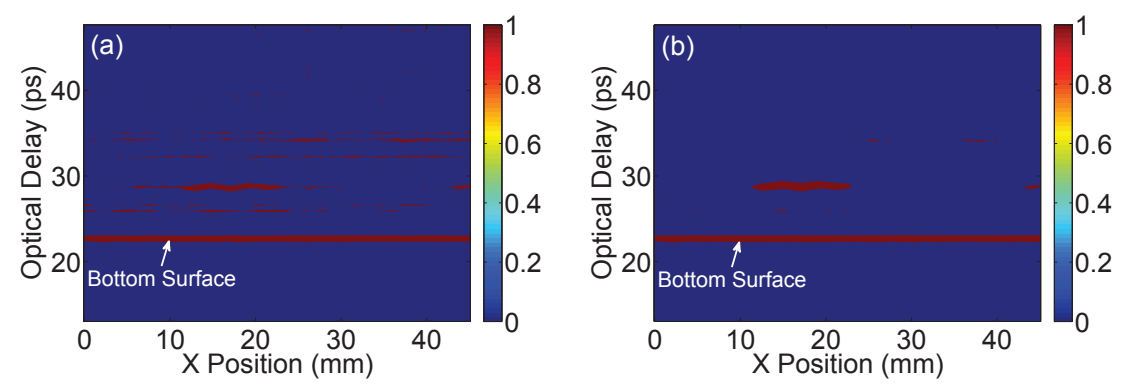

Fig. 11 Binary $\mathrm{THz}$ B-scan images based on $\mathrm{THz}$ waveforms (a) without and (b) with wavelet de-noising.

THz B-scans without and with wavelet de-noising are shown in Fig. 10. Fluctuations in the original $\mathrm{THz}$ waveforms lead to the multiple horizontal lines in the THz B-scan in Fig. 10(a). These horizontal lines may be misinterpreted as internal layers in the samples. By applying wavelet de-noising, the horizontal artifacts associated with water vapor are suppressed, shown in Fig. 10(b). Again, we apply the $50 \%$ rule to obtain binary THz B-scan images. In this case, we assign the highest value within the time slice between 27 and 29 ps as $100 \%$, then color all pixels red that have a value of at least $50 \%$ the max- 
imum. The binary $\mathrm{THz}$ B-scans based on the data without and with wavelet de-noising are shown in Fig. 11, which also shows that the binary $\mathrm{THz}$ B-scan based on processed data provides better image to locate the delamination in depth.

\section{Conclusion}

A woven glass fiber-reinforced composite laminate with forced delamination is characterized by reflective $\mathrm{THz}$ imaging. The thickness of the forced delamination is about $25 \mu \mathrm{m}$, which is below the axial resolution in our $\mathrm{THz}$ scanning. Fluctuations due to the ambient water vapor, which cannot be cancelled by averaging, substantially lower the contrast in $\mathrm{THz} \mathrm{C}$ - and B-scans. Enhanced $\mathrm{THz} \mathrm{C}$ - and B-scans are obtained by applying wavelet shrinkage de-noising to the reflected $\mathrm{THz}$ waveforms for each pixel. Wavelet shrinkage de-noising is shown in this case effectively to remove the fluctuations due to the water vapor while preserving the features associated with the thin delamination, thus providing significantly clearer $\mathrm{THz}$ images for locating and sizing the small delamination in these materials in three dimensions.

This approach is of great interest in cases where undesired features associated with atmospheric water vapor of other material resonances may obscure features to be detected. In view of the difficulty and expense in purging with dry nitrogen to physically remove the water-vapor features in many cases, the demonstrated results will be of great interest for a range of laboratory- and field-based $\mathrm{THz}$ NDE applications.

Acknowledgements The authors acknowledge R. Bergman of Ten Cate Advanced Composites BV for kindly supplying the samples in this study and for helpful discussions. The authors also acknowledge the financial support of the Conseil Régional de Lorraine, and the Fonds Européen de Développement Régional (FEDER)

\section{References}

1. F Rutz, M Koch, S Khare, M Moneke, H Richter and U Ewert, J INFRARED MILLIM TE 27, 547-56(2002).

2. I Amenabar, F Lopez and A Mendikute, J INFRARED MILLIM TE 34, 152-69(2013).

3. C D Stoik, M J Bohn and J L Blackshire, OPT EXPRESS 16, 17039-51(2008).

4. C D Stoik, M J Bohn and J L Blackshire, NDT \& E INT 43, 106-15(2010).

5. J Dong, B Kim, A Locquet, P McKeon, N Declercq and D S Citrin, COMPOS PART B-ENG 79, 667-75(2015)

6. F Ospald, W Zouaghi, R Beigang, C Matheis, J Jonuscheit, B Recur, J-P Guillet, P Mounaix, W Vleugels, P V Bosom, L V González, I López, R M Edo, OPT ENG 53, 031208-08(2013).

7. K Naito, Y Kagawa, S Utsuno, T Naganuma and K Kurihara, COMPOS SCI TECHNOL 69, 2027-29(2009)

8. C Jördens, M Scheller, S Wietzke, D Romeike, C Jansen, T Zentgraf, K Wiesauer, V Reisecker and M Koch, COMPOS SCI TECHNOL 70, 472-77(2010).

9. N Palka and D Miedzinska, OPT QUANT ELECTRON 46, 515-25(2014).

10. N Palka, R Panowicz, F Ospald and R Beigang, J INFRARED MILLIM TE 36, 77088(2015). 
11. V I Bezborodov, V K Kiseliov, O S Kosiak, Y M Kuleshov, P K Nesterov and M S Yanovsky, TELECOMM RADIO ENG+ 73, 83-93(2014).

12. J Dong, A Locquet, N Declercq and D S Citrin, Polarization-resolved terahertz imaging of intra- and inter-laminar damages in hybird fiber-reinforced composites laminate subject to low-velocity impact. Submitted.

13. D Mittleman, Sensing with terahertz radiation.(Springer-Verlag Berlin Heidelberg GmbH, 2003), pp. 40-43.

14. W Withayachumnankul, B M Fischer and D Abbott, P ROY SOC A-MATH PHY 464, 2435-56(2008).

15. Y Wang, Z Zhao, Z Chen, K Kang, B Feng and Y Zhang, J APPL PHYS 102, 113108(2007).

16. C Ryu and S G Kong, APPL OPTICS 49, 927-35(2010).

17. Y Chen, Y Sun and E Pickwell-Macpherson, FLUCT NOISE LETT 9, 387-94(2010).

18. M Schwerdtfeger, E Castro-Camus, K Krügener, W Viöl and M Koch, APPL OPTICS $52,375-80(2013)$.

19. D M Mittleman, R H Jacobsen, R Neelamani, R G Baraniuk and M C Nuss, APPL PHYS B-LASERS O 67, 379-90(1998).

20. Y Chen, S Huang and E Pickwell-MacPherson, OPT EXPRESS 18, 1177-90(2010).

21. B Ferguson and D Abbott, MICROELECTR J 32, 943-53(2001).

22. T Yasuda, T Iwata, T Araki and T Yasui, APPL OPTICS 46, 7518-26(2007).

23. R A Smith, L D Jones, S J Willsher and A B Marriott, INSIGHT 40, 44-49(1998). 